\title{
Reprogramming based gene therapy for inherited red blood cell disorders
}

\author{
Xiuling $\mathrm{Xu}^{1,{ }^{*}}$, Jing $\mathrm{Qu}^{1,2,{ }^{*}}$, Keiichiro Suzuki ${ }^{2}, \mathrm{Mo} \mathrm{Li}^{2}$, Weizhou Zhang ${ }^{4}$, Guang-Hui Liu ${ }^{1}$, \\ Juan Carlos Izpisua Belmonte ${ }^{2,3}$
}

\begin{abstract}
${ }^{I}$ National Laboratory of Biomacromolecules, Institute of Biophysics, Chinese Academy of Sciences, Beijing 100101, China; ${ }^{2}$ Gene Expression Laboratory, Salk Institute for Biological Studies, 10010 North Torrey Pines Road, La Jolla, CA 92037, USA; ${ }^{3}$ Center for Regenerative Medicine in Barcelona, Dr. Aiguader 88, 08003 Barcelona, Spain; ${ }^{4}$ Laboratory of Gene Regulation and Signal Transduction, Department of Pharmacology, School of Medicine, University of California, San Diego, La Jolla, CA 92093, USA Cell Research (2012) 22:941-944. doi:10.1038/cr.2012.54; published online 3 April 2012
\end{abstract}

Hematopoietic stem cell transplantation remains the only curative therapy for certain genetic diseases of the hematopoietic system, including some inheritable deficiencies in red blood cells (RBC). Recent advances in induced pluripotent stem cells may open a new era for the cure of such severe genetic RBC diseases.

Red blood cells (RBCs) are unique, highly specialized, and the most abundant cells in humans and other organisms. The primary function of RBCs is transportation of the respiratory gases $\mathrm{O}_{2}$ and $\mathrm{CO}_{2}$. Meanwhile, RBCs are also the main antioxidant reservoir for the whole body. Dysfunction of RBCs very often leads to anemia and can be caused by various factors, including different pathologic conditions and inherited genetic mutations [1]. Many anemic patients require life-long blood transfusions and iron-chelating therapies, which have severe side effects and add a significant burden to the health

*These two authors contributed equally to this work.

Correspondence: Guang-Hui Liu ${ }^{a}$, Weizhou

Zhang $^{\mathrm{b}}$, Juan Carlos Izpisua Belmonte ${ }^{\mathrm{c}}$

${ }^{\mathrm{a} E}$-mail: ghliu@ibp.ac.cn

bE-mail: w4zhang@ucsd.edu

‘E-mail: belmonte@salk.edu, izpisua@cmrb.eu care system. Currently, the only curative therapy for certain genetic RBC diseases is hematopoietic stem cell (HSC) transplantation from Human Leukocyte Antigen (HLA)-matched donors or gene-corrected autologous HSCs. Although HSC transplantation remains the most attractive and promising treatment for hematologic diseases, there are still obstacles, including difficulties in finding matched donors, in vitro expansion of $\mathrm{HSC}$, low efficiency of gene delivery/correction, and safety concerns due to random viral vector integration into the host genome. Induced pluripotent stem cells (iPSCs) provide a new promising strategy for cell therapy of RBC diseases [2] (Figure 1). They show unlimited proliferative ability in culture allowing production of sufficient transplantable cells, can be used in an autologous setting and are amenable to gene editing for correction of disease mutations. iPSCs, together with novel technologies like lineage conversion, may constitute, in the near future, a valid alternative to HSCs for the treatment of anemic patients.

Thalassemia is a group of hemoglobinopathies caused by mutations in the globin genes, which usually lead to functional loss or reduction of globin protein expression. $\beta$-thalassemia, a type of progressive anemia caused by the deficiency of $\beta$-globin chain $(H B B)$ expression during fetal to adult transition [3], is common worldwide. $\beta$-thalassemia patients have genetic mutations (point substitutions or small deletions) in the $H B B$ gene. Ye and colleagues [3] reported the first case of producing human iPSCs from a $\beta$-thalassemia patient who was homozygous for a four-base pair (bp) loss-of-function deletion (CTTT) in codon 41/42. Patient-derived fibroblasts were reprogrammed to iPSCs using the four Yamanaka factors (OCT4, SOX2, KLF4, c-MYC) and then differentiated into hematopoietic-like colonies, in which hemoglobinized erythroid cells were observed as characterized by Giemsa staining and hemoglobin staining. Retroviruses were used to deliver the reprogramming transcriptional factors, which may raise safety concerns due to random viral integration into the host genome. In a separate study, the Gao lab reprogrammed fibroblasts from a $\beta$-thalassemia patient with homozygous 41/42-4 bp CTTT deletion in the $H B B$ gene using three transcriptional factors (OCT4, SOX2, KLF4) [4, 5]. Of note, they also corrected the genetic mutation in patient iPSCs by a specific gene editing technique based on homologous re- 


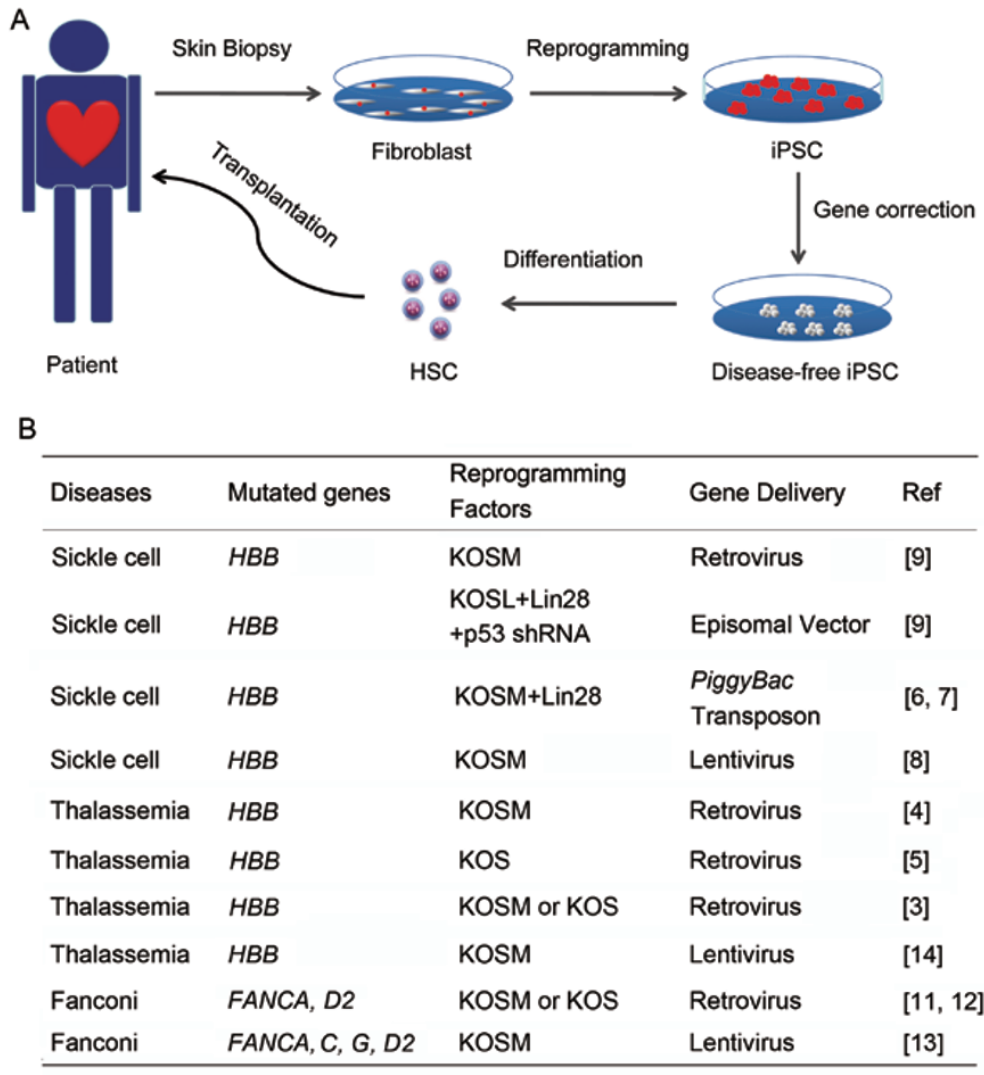

Figure 1 (A) iPSC-based cell therapy for inheritable RBC disorders. Patient fibroblasts can be obtained from skin biopsy or other tissues, followed by reprogramming with distinct set of factors to establish patient-specific iPSCs. These iPSCs bearing genetic mutations will be corrected with gene editing approaches to produce disease-free iPSCs. The corrected iPSCs are autologous and able to be differentiated into hematopoietic stem cells in vitro for therapeutic purposes. (B) Summary of iPSC from anemic patients. K, KLF4; O, OCT4; S, SOX2; L, L-MYC; M, C-MYC.

combination. The corrected iPSC lines exhibited similar differentiation ability towards $\mathrm{CD} 34^{+}$hematopoietic progenitor cells (HPCs) to their wild-type counterparts, based on an OP9 stromal cells co-culture system. The $\mathrm{CD} 34^{+}$cells differentiated from patient-derived iPSCs or corrected iPSCs were enriched, and their differentiation ability was analyzed by colony forming assay in vitro. The colonies formed by $\mathrm{CD} 34^{+}$cells differentiated from patient-derived iPSCs or corrected iPSCs exhibited similar morphology and multilineage potential. In vivo differentiation was performed by intra-tibial transplantation of CD $34^{+}$ HPCs into irradiated SCID mice. The transplanted cells were indeed functional and capable of undergoing erythroid differentiation in vivo. Human fetal globin ( $\gamma$-globin) expression could be detected in all transplanted mice, while the adult globin ( $\beta$-globin) expression was only detected in mice transplanted with the derivatives from hESCs and the corrected iPSCs, but not from the uncorrected ones. Whole blood analyses from transplanted animals revealed elevated human hemoglobin levels in corrected iPSC-derived CD $34^{+}$HPC recipients relative to mice receiving non-corrected $\mathrm{CD} 34^{+}$HPCs one week after transplantation. The difference between different groups diminished 4 weeks post transplantation [5]. These results indicate that transplanted $\mathrm{CD} 34^{+}$ cells bear limited erythropoietic potential in transplanted SCID mice.

Sickle cell disease is a severe genetic disease caused by a point mutation (GAG to GTG) at codon 6 of the human $H B B$ gene, resulting in a defective form of adult hemoglobin. RBCs from sickle cell disease patients can clog blood vessels, causing pain, fatigue, infection, organ damage, and even premature death. The Cheng lab built a iPSC line from bone marrow stromal cells of a patient using a single piggybac transposon vector expressing 4-5 transcription factors $[6,7]$. In this case, zinc finger nuclease technology was employed to make a specific double-strand break to stimulate homologous recombination near the mutation site of $\beta^{s}$ alleles [6, 7]. One iPSC line with single corrected allele was obtained and able to be differentiated into nucleated erythroblasts rather than erythrocytes in vitro, probably due to limitations of current differentiation protocol. Fetal globin $(H B G)$ was the most highly expressed hemoglobin in these erythroblasts, whereas adult $H B B$ transcripts were detectable by PCR at a relatively low level [8]. Our group also has efficiently generated gene-corrected sickle cell disease patient cells from integration-free iPSCs by combining episomal vectors-based reprogramming and helper-dependent adenoviral vector (HDAdV)-mediated gene correction [9]. Moreover, we have previously demonstrated that HDAdVmediated gene editing did not result in genetic and epigenetic abnormalities [10] . Our results indicate that one $H B B$ gene-correction HdAdV has the ability to correct any mutation located in $H B B$, therefore it may be suitable for other forms of $\beta$-globinopathies.

Fanconi anemia (FA) is a complex genetic blood disorder and a rare aplastic anemia due to the failure of bone marrow that stops making or does not make enough bone marrowderived blood cells including RBCs. 
FA is caused by mutations in any of 14 genes in the FA pathway. FA cells are very sensitive to DNA damage due to dysfunctions in repairing doublestranded DNA breaks. Interestingly, attempts to reprogram FA fibroblasts or keratinocytes were unsuccessful unless genetic defects were complemented via lentiviral gene delivery. One exception occurred in a patient with a hypomorphic defect in FANCD2 where patient-derived fibroblasts were able to be reprogrammed without gene correction, though the reprogrammed cells could not be maintained for more than three passages. The resistance of FA fibroblasts to reprogramming indicates that an intact FA pathway is required for somatic cell reprogramming [11, 12]. In contrast, gene-corrected iPSCs restored the function of FA pathway, and were thus refractory to reprogramming-associated DNA damage. In vitro differentiation towards hematopoietic lineages resulted in similar morphologies and proportions of erythroid and myeloid colonies in both gene-corrected iPSCs and normal iPSCs [11]. Recently, the Daley lab has extended these results. They have demonstrated increased FANCD2 foci on chromosomes upon reprogramming, indicating that the FA pathway is activated in this process. Of note, increased reactive oxygen species (ROS) is a critical contributor to the resistance in reprogramming FA fibroblasts, as FA fibroblasts could be reprogrammed in the presence of antioxidant $\mathrm{N}$-actylecysteine or under hypoxic conditions $\left(5 \% \mathrm{O}_{2}\right)$, but at a much lower efficiency than normal or corrected fibroblasts. HPCs derived from the FA-iPSCs were capable of differentiation into several blood cell lineages in vitro based on colony forming assay [13].

Besides in situ gene correction for pathogenic mutations, expression of wild-type genes remains a good option for rescuing disease phenotypes. To ensure cell therapy with minimal undesired mutagenesis caused by random viral integrations, Papapetrou et al. [14] have proposed 5 criteria to reduce oncogenic potential of genome-integrated exogenous transgenes. The authors applied these rules to identify safely corrected iPSCs from $\beta$-thalassemia patient-derived iPSCs [14]. Patient iPSCs were transduced with viral vectors expressing $\beta$-globin at a low multiplicity of infection (MOI), and then iPSC lines harboring a single copy of $\beta$-globin from a single viral integration were isolated. 15 iPSC lines with single viral integrations were randomly picked up, only one of which met all five criteria. When differentiated to erythrocytes, the expression of vector-encoded $\beta$-globin reached about $85 \%$ of normal $\beta$-globin levels. The five criteria in guiding selection of relatively safe patient-specific iPSCs provide an alternative strategy for loss-of-function genetic diseases and will also be applicable to other anemic diseases mentioned above.

In summary, generation of RBCs from patient-specific iPSCs is not only exciting but also holds great potential for treating genetic blood diseases. The erythroid progeny of iPSC lines preferentially express embryonic and fetal globins like $\alpha$-, $\varepsilon^{-}$and $\gamma$-globin, but not the adult $\beta$-globin [15-17]. Thus, the switch from primitive to definitive erythropoiesis remains an obstacle for iPSC-based cell therapy for $\beta$-globin disorders such as $\beta$-thalassemia and sickle cell disease. Recently, the development of trans-differentiation methodologies provides alternative options to iPSC-based approaches. In this regard, the Bhatia group reported direct conversion of human dermal fibroblasts to hematopoietic lineages without forcing cells to an iPSC state [18], which will reduce tumorigenic risk relative to iPSC-based therapy. $\mathrm{CD}_{4} 5^{+}$cells directly derived from human dermal fibroblasts expressing OCT4 $\left(\mathrm{CD}^{2} 5^{+} \mathrm{Fibs}^{\mathrm{OCT} 4}\right)$ were able to be differentiated into erythroid lineage upon erythropoietin (EPO) stimulation, leading to both primitive and mature erythrocyte morphologies. In agreement, EPO-treated $\mathrm{CD} 45^{+} \mathrm{Fibs}^{\mathrm{OCT} 4}$ cells exhibited increased expression of adult $\beta$-globin protein, modest level of fetal globins, and complete loss of embryonic globins. It will be of great importance to improve trans-differentiation protocols in order to obtain pure and functional blood cells suitable for future clinical transplantation. In addition, developing efficient gene editing tools amenable for manipulating somatic cells represents another challenge for cell-based therapies of genetic diseases.

\section{Acknowledgments}

GHL is supported by 100 Talents Program of Chinese Academy of Sciences, and a Glenn Aging Foundation grant. JQ was partially supported by an AFAR/Ellison Medical Foundation postdoctoral fellowship. Work in the laboratory of JCIB was supported by Sanofi-Aventis, The Helmsley Charitable Trust and The Ellison Medical Foundation. WZ is supported by NIH Pathway to Independence award (K99/R00 CA158055-01).

\section{References}

1 Loustau V, Guillaud C, Garcon L, Godeau B, Michel M. Hemolytic anemia in adults: Main causes and diagnostic procedure. Presse Med 2011; 40:470485.

2 Douay L. In vitro generation of red blood cells for transfusion: a model for regenerative medicine. Regen Med 2012; 7:1-2.

3 Ye L, Chang JC, Lin C, Sun X, Yu J, Kan YW. Induced pluripotent stem cells offer new approach to therapy in thalassemia and sickle cell anemia and option in prenatal diagnosis in genetic diseases. Proc Natl Acad Sci USA 2009; 106:9826-9830.

4 Wang Y, Jiang Y, Liu S, Sun X, Gao S. Generation of induced pluripotent stem cells from human beta-thalassemia fibroblast cells. Cell Res 2009; 19:11201123.

5 Wang Y, Zheng CG, Jiang Y, et al. Genetic correction of beta-thalassemia patient-specific iPS cells and its use in improving hemoglobin production in irradiated SCID mice. Cell Res 2012; 22:637-648.

6 Zou J, Mali P, Huang X, Dowey SN, 
Cheng L. Site-specific gene correction of a point mutation in human iPS cells derived from an adult patient with sickle cell disease. Blood 2011; 118:45994608.

7 Mali P, Chou BK, Yen J, et al. Butyrate greatly enhances derivation of human induced pluripotent stem cells by promoting epigenetic remodeling and the expression of pluripotency-associated genes. Stem Cells 2010; 28:713-720.

8 Sebastiano V, Maeder ML, Angstman $\mathrm{JF}$, et al. In situ genetic correction of the sickle cell anemia mutation in human induced pluripotent stem cells using engineered zinc finger nucleases. Stem Cells 2011; 29:1717-1726.

9 Li M, Suzuki K, Qu J, et al. Efficient correction of hemoglobinopathy-causing mutations by homologous recombination in integration-free patient iPSCs. Cell Res 2011; 21:1740-1744.

10 Liu GH, Suzuki K, Qu J, et al. Tar- geted gene correction of laminopathyassociated LMNA mutations in patientspecific iPSCs. Cell Stem Cell 2011; 8:688-694.

11 Raya A, Rodriguez-Piza I, Guenechea $\mathrm{G}$, et al. Disease-corrected haematopoietic progenitors from Fanconi anaemia induced pluripotent stem cells. Nature 2009; 460:53-59.

12 Raya A, Rodriguez-Piza I, Navarro S, et al. A protocol describing the genetic correction of somatic human cells and subsequent generation of iPS cells. Nat Protoc 2010; 5:647-660.

13 Muller LU, Milsom MD, Harris CE, et al. Overcoming reprogramming resistance of fanconi anemia cells. Blood 2012 Feb 27. doi:10.1182/ blood-2012-02-408674

14 Papapetrou EP, Lee G, Malani N, et al. Genomic safe harbors permit high beta-globin transgene expression in thalassemia induced pluripotent stem cells. Nat Biotechnol 2011; 29:73-78.

15 Chang KH, Nelson AM, Cao H, et al. Definitive-like erythroid cells derived from human embryonic stem cells coexpress high levels of embryonic and fetal globins with little or no adult globin. Blood 2006; 108:1515-1523.

16 Qiu C, Olivier EN, Velho M, Bouhassira EE. Globin switches in yolk saclike primitive and fetal-like definitive red blood cells produced from human embryonic stem cells. Blood 2008; 111:2400-2408.

17 Chang KH, Huang A, Hirata RK, Wang PR, Russell DW, Papayannopoulou T. Globin phenotype of erythroid cells derived from human induced pluripotent stem cells. Blood 2010; 115:25532554.

18 Szabo E, Rampalli S, Risueno RM, et al. Direct conversion of human fibroblasts to multilineage blood progenitors. Nature 2010; 468:521-526. 